IJMMS 25:5 (2001) 299-304

PII. S0161171201005051

http://ijmms.hindawi.com

(c) Hindawi Publishing Corp.

\title{
STRUCTURE OF WEAKLY PERIODIC RINGS WITH POTENT EXTENDED COMMUTATORS
}

\author{
ADIL YAQUB \\ (Received 1 July 1999 and in revised form 3 May 2000) \\ Dedicated to the memory of Professor Hisao Tominaga
}

\begin{abstract}
A well-known theorem of Jacobson (1964, page 217) asserts that a ring $R$ with the property that, for each $x$ in $R$, there exists an integer $n(x)>1$ such that $x^{n(x)}=x$ is necessarily commutative. This theorem is generalized to the case of a weakly periodic ring $R$ with a "sufficient" number of potent extended commutators. A ring $R$ is called weakly periodic if every $x$ in $R$ can be written in the form $x=a+b$, where $a$ is nilpotent and $b$ is "potent" in the sense that $b^{n(b)}=b$ for some integer $n(b)>1$. It is shown that a weakly periodic ring $R$ in which certain extended commutators are potent must have a nil commutator ideal and, moreover, the set $N$ of nilpotents forms an ideal which, in fact, coincides with the Jacobson radical of $R$.
\end{abstract}

2000 Mathematics Subject Classification. Primary 16U80, 16D70.

1. Introduction. A ring $R$ is called periodic if for each $x$ in $R$ there exist distinct positive integers $m$ and $n$ such that $x^{m}=x^{n}$. An element $x$ is called potent if for some integer $n=n(x)>1, x^{n}=x . R$ is called weakly periodic if every $x$ in $R$ can be written (not necessarily uniquely) as a sum of a nilpotent element and a potent element. It is well known that a periodic ring is necessarily weakly periodic (see [2]). Whether a weakly periodic ring is necessarily periodic is apparently not known. Moreover, by a theorem of Chacron (see [3]), $R$ is periodic if and only if for each $x$ in $R$, there exists a positive integer $k=k(x)$ and a polynomial $f(\lambda)=f_{x}(\lambda)$ with integer coefficients such that $x^{k}=x^{k+1} f(x)$. For $x, y$ in $R,[x, y]_{1}=[x, y]=x y-y x$ denotes the usual commutator, and for every positive integer $k>1$, we define the extended commutator $[x, y]_{k}$ inductively by $[x, y]_{k}=\left[[x, y]_{k-1}, y\right]$.

2. Main results. We begin with some basic facts about weakly periodic rings.

LEMMA 2.1. The homomorphic image of any weakly periodic ring is weakly periodic.

This follows readily from the definition of a weakly periodic ring.

LEMMA 2.2. A weakly periodic division ring is necessarily commutative.

This follows from the " $x^{n(x)}=x$ " theorem of Jacobson (see [5]).

LEMmA 2.3. Let $R$ be a weakly periodic ring, $N$ the set of nilpotents, and $J$ the Jacobson radical of $R$. Then $J \subseteq N$. 
Proof. Suppose $j \in J$. Then,

$$
j=a+b, \quad a \in N, \quad b^{n}=b \quad \text { for some } n>1 .
$$

Suppose $a^{q}=0$. Then,

$$
(j-b)^{(n-1) q+1}=a^{(n-1) q+1}=0,
$$

which implies (since $j \in J$ ) $b^{(n-1) q+1} \in J$. But $b^{(n-1) q+1}=b$, since $b^{n}=b$, and hence $b \in J$. Since $b^{n-1}$ is an idempotent element in $J, b^{n-1}=0$. Therefore, $b=b^{n}=0$, and hence by (2.1), $j=a \in N$. Thus, $J \subseteq N$.

THEOREM 2.4. Let $R$ be a weakly periodic ring and suppose that $N$ is the set of nilpotents of $R$. Let $n>1$ be a fixed integer. Suppose that for all $x_{1}, \ldots, x_{n}$ in $R \backslash N, \sigma$ is a permutation in $S_{n}$ such that $\sigma(n) \neq n$. Suppose, further, that for all $x_{1}, \ldots, x_{n}$ in $R \backslash N$, there exists a positive integer $k$ such that the following extended commutator is potent, namely

$$
\left[x_{1} \cdots x_{n}, x_{\sigma(1)} \cdots x_{\sigma(n)}\right]_{k} \text { is potent, } \quad \forall x_{i} \in R \backslash N .
$$

Then,

(i) The commutator ideal of $R$ is nil, $(C(R) \subseteq N)$.

(ii) $N$ is an ideal of $R$.

(iii) $N=J$, the Jacobson radical of $R$.

(iv) $R$ is periodic.

Proof. The semisimple ring $R / J$ is isomorphic to a subdirect sum of primitive rings $R_{i}(i \in \Gamma)$. By Lemma 2.1, each $R_{i}$ is a weakly periodic ring. Now, by Jacobson's density theorem, we must have

(a) $R_{i}$ is a division ring, or

(b) for some positive integer $m>1$, there exists a subring $T_{i}$ of $R_{i}$ which maps homomorphically onto $D_{m}$, the complete matrix ring of $m \times m$ matrices over some division ring $D$.

In case (a), $R_{i}$ is commutative, by Lemma 2.2. In case (b), $D_{m}$ must satisfy (2.3), since (2.3) is inherited by all subrings and all homomorphic images of $R$, where $m>1$. The net result is:

(*) The ring $D_{m}$ of all $m \times m$ matrices over the division ring $D$ satisfies (2.3), where $m>1$.

That statement $(*)$ is false can be seen by taking

$$
x_{1}=x_{2}=\cdots=x_{n-1}=E_{11}, \quad x_{n}=E_{11}+E_{12} .
$$

In verifying this, note that

$$
\begin{gathered}
x_{1} \cdots x_{n}=E_{11}\left(E_{11}+E_{12}\right)=E_{11}+E_{12} \\
x_{\sigma(1)} \cdots x_{\sigma(n)}=\left(E_{11}+E_{12}\right) E_{11}=E_{11} \\
{\left[x_{1} \cdots x_{n}, x_{\sigma(1)} \cdots x_{\sigma(n)}\right]=-E_{12}} \\
{\left[x_{1} \cdots x_{n}, x_{\sigma(1)} \cdots x_{\sigma(n)}\right]_{k}=(-1)^{k} E_{12},}
\end{gathered}
$$

which is not potent. This contradiction shows that case (b) never occurs, and hence 
each $R_{i}$ is a commutative division ring (case (a)). Thus, $R / J$ is indeed commutative, and hence

$$
C(R) \subseteq J, \quad(C(R) \text { denotes the commutator ideal of } R) .
$$

Combining this with Lemma 2.3, we see that $C(R) \subseteq N$, which proves (i). Part (ii) follows at once from part (i) by considering the commutative ring $R / C(R)$. To prove (iii), observe that $N \subseteq J$ (since $N$ is an ideal) while $J \subseteq N$, by Lemma 2.3. Thus, $N=J$.

To prove part (iv), let $x \in R$. Then, by definition of weakly periodic ring, there exist elements $a$ and $b$ such that

$$
x=a+b, \quad a \in N ; \quad b^{\gamma}=b, \quad \text { for some } \gamma>1 .
$$

Hence, since $N$ is an ideal (part (ii)) and $a \in N$,

$$
x-a=b=b^{\gamma}=(x-a)^{\gamma}=x^{\gamma}+a_{0} \quad\left(a_{0} \in N\right) .
$$

Therefore, $x-x^{\gamma}=a+a_{0} \in N$, and thus $\left(x-x^{\gamma}\right)^{\alpha}=0$ for some positive integer $\alpha$. Hence, $x^{\alpha}=x^{\alpha+1} f(x), f(\lambda) \in \mathbb{Z}[\lambda]$, and thus by Chacron's theorem (see Section 1) $R$ is periodic. This proves the theorem.

In preparation for the proof of the next theorem, we first prove the following lemmas.

LEMMA 2.5. Let $R$ be an arbitrary ring (not necessarily weakly periodic), and suppose $N$ is the set of nilpotents of $R$. Let $n>1$ be a fixed integer. Suppose that for all $x_{1}, \ldots, x_{n}$ in $R \backslash N, \sigma$ is a permutation in $S_{n}$ such that $\sigma(1) \neq 1$ and $\sigma(n) \neq n$. Suppose, further, that for all $x_{1}, \ldots, x_{n}$ in $R \backslash N$, there exists a positive integer $k$ such that

$$
\left[x_{1} \cdots x_{n}, x_{\sigma(1)} \cdots x_{\sigma(n)}\right]_{k} \text { is potent, } \quad \forall x_{i} \in R \backslash N .
$$

Then, the set $E$ of idempotents of $R$ is central.

Proof. Suppose $e \in E, x \in R, a=e x-e x e, f=e+a$. We claim that $e f=f e$. Suppose $e f \neq f e$, then $e \neq 0, f \neq 0$, and (since $e^{2}=e, f^{2}=f$ ) hence $e \notin N, f \notin N$. Therefore, by (2.9) with $x_{1}=\cdots=x_{n-1}=e, x_{n}=f$, we have $[e f, f e]_{k}$ is potent, and hence $[f, e]_{k}=(-1)^{k} a$ is potent. Since $a^{2}=0$, it follows that $a=0$. Hence, $f=e+a=e$, which contradicts the hypothesis $e f \neq f e$. This contradiction shows that $e f=f e$, and hence $e(e+a)=(e+a) e$. Thus, $a=e a=a e=0$. Hence, $e x=e x e$. A similar argument, using $a^{\prime}=x e-e x e, f^{\prime}=e+a^{\prime}$ shows that $x e=e x e$, and hence $e x=x e$. This proves the lemma.

LEMMA 2.6. Suppose that $R$ is a weakly periodic ring which satisfies the hypotheses of Theorem 2.4. Suppose $\delta: R \rightarrow R^{*}$ is a homomorphism of $R$ onto $R^{*}$, and let $N$ be the set of nilpotents of $R$. Then, the set $N^{*}$ of nilpotents of $R^{*}$ coincides with the set $\delta(N)$.

Proof. By Theorem 2.4(iv), $R$ is periodic. The lemma now follows from [1].

LEMMA 2.7. Let $R$ be a subdirectly irreducible ring. Then, the only central idempotents of $R$ are 0 and 1 (if $1 \in R$ ).

This lemma is well known, and we omit the proof. 
LEMmA 2.8. Let $R$ be a ring, and let $x, y \in R$. Suppose that $[x, y]$ commutes with $x$. Then, for all positive integers $k$, we have

$$
\left[x^{k}, y\right]=k x^{k-1}[x, y] .
$$

(Equivalently, $\left.\left[y, x^{k}\right]=k x^{k-1}[y, x].\right)$

This follows at once, by induction.

LEMMA 2.9. Let $R$ be a periodic ring with the set $N$ of nilpotents commutative. If for each $a \in N$ and $x \in R$ there exists a positive integer $k$ such that $[a, x]_{k}=0$, then $R$ is commutative.

This lemma was proved by Bell [4].

We are now in a position to prove our next theorem.

THEOREM 2.10. Let $R$ be a weakly periodic ring, and let $N$ denote the set of nilpotents of $R$. Let $n>1$ be a fixed integer. Suppose that for all $x_{1}, \ldots, x_{n}$ in $R \backslash N, \sigma$ is a permutation in $S_{n}$ such that $\sigma(1)=n$ and $\sigma(n)=1$. Suppose that, for all $x_{1}, \ldots, x_{n}$ in $R \backslash N$, there exists a positive integer $k$ such that

$$
\left[x_{1} \cdots x_{n}, x_{\sigma(1)} \cdots x_{\sigma(n)}\right]_{k} \text { is potent, } \quad \forall x_{i} \in R \backslash N \text {. }
$$

Suppose, further, that

$$
[a, b] \text { is potent } \forall a, b \in N \text {. }
$$

Then, $R$ is commutative.

Proof. In view of Lemma 2.6, all the hypotheses are inherited by homomorphic images of $R$; and since every ring is isomorphic to a subdirect sum of subdirectly irreducible rings, we may assume that $R$ is subdirectly irreducible. Since $N$ is an ideal, by Theorem 2.4(ii), we see that for all $a, b$ in $N,[a, b]$ is both potent (see (2.12)) and nilpotent, and hence $[a, b]=0$, which implies that $N$ is commutative.

Now, $R$ is periodic, by Theorem 2.4(iv), and hence some power of each element of $R$ is idempotent. Therefore, by Lemmas 2.5 and 2.7 , either $R$ is nil or $R$ has an identity 1 . In the first case, $R=N$ is commutative and there is nothing further to prove. So we assume that $1 \in R$.

Let $a \in N$ and $x \in R \backslash N$. Since $1+a \notin N$, there exists a positive integer $k$ such that

$$
\begin{aligned}
& {[(1+a) \cdot 1 \cdot 1 \cdots 1 \cdot x, x \cdot 1 \cdot 1 \cdots 1 \cdot(1+a)]_{k} \text { is potent, }} \\
& \text { and thus }[x+a x, x+x a]_{k} \text { is potent. }
\end{aligned}
$$

Next, we show, by induction, that

$$
[x+a x, x+x a]_{m}=[a, x]_{m+1} \text { for all positive integers } m \text {. }
$$

To begin with, observe that

$$
[x+a x, x+x a]_{1}=[x, x a]+[a x, x]+[a x, x a] .
$$


Since $N$ is a commutative ideal of $R$ and $a \in N$, therefore $[a x, x a]=0$, and hence (2.15) is equivalent to

$$
[x+a x, x+x a]_{1}=-[x a, x]+[a x, x]=[a, x]_{2} .
$$

Hence (2.14) is true for $m=1$. Now, suppose (2.14) is true for $m=q$. That is, suppose that

$$
[x+a x, x+x a]_{q}=[a, x]_{q+1} .
$$

This induction hypothesis implies that

$$
\begin{aligned}
{[x+a x, x+x a]_{q+1} } & =\left[[a, x]_{q+1}, x+x a\right] \\
& =\left[[a, x]_{q+1}, x\right]+\left[[a, x]_{q+1}, x a\right] \\
& =\left[[a, x]_{q+1}, x\right]=[a, x]_{q+2},
\end{aligned}
$$

since $\left[[a, x]_{q+1}, x a\right]=0$ (recall that $a \in N$ and $N$ is a commutative ideal of $R$ ). Thus, (2.14) is true for $m=q+1$, completing this induction proof of (2.14). Now, combining (2.13) and (2.14), we see that

$$
[a, x]_{k+1} \text { is potent }(a \in N, x \in R) .
$$

Since $[a, x]_{k+1}$ is also in the ideal $N$, therefore this extended commutator is both nilpotent and potent, and hence

$$
[a, x]_{k+1}=0 .
$$

Keeping in mind that $R$ is periodic and $N$ is commutative, and combining (2.19) with Lemma 2.9, it follows that $R$ is commutative, and the theorem is proved.

COROLLARY 2.11. Suppose $R$ is a periodic ring with commuting nilpotents and with the property that, for all $x, y$ in $R$, there exists a positive integer $k$ such that $[x y, y x]_{k}=0$. Then, $R$ is commutative.

Proof. Since $R$ is periodic, $R$ is also weakly periodic (see Section 1). Therefore, all the hypotheses of Theorem 2.10 are satisfied (take $n=2$ in (2.11)), and hence $R$ is commutative.

The following is another corollary which yields a result proved by Putcha and Yaqub [6].

COROLLARY 2.12. A periodic ring with commuting nilpotents and central commutators is commutative.

Proof. Let $x, y$ be any elements of $R$. Since $[x, y]$ is in the center of $R$, therefore $[[x, y], y]=0$; that is, $[x, y]_{2}=0$. Thus,

$$
[x, y]_{2}=0 \quad \forall x, y \in R .
$$

In particular, $[x y, y x]_{2}=0$, and the corollary now follows by taking $k=2$ in Corollary 2.11. 
ACKNOWLEDGEMENT. I would like to thank the referee for the valuable suggestions made, which resulted in improving the paper.

\section{REFERENCES}

[1] H. E. Bell, Some commutativity results for periodic rings, Acta Math. Acad. Sci. Hungar. 28 (1976), no. 3-4, 279-283. MR 54\#7556. Zbl 335.16035.

[2] _ A commutativity study for periodic rings, Pacific J. Math. 70 (1977), no. 1, 29-36. MR 58\#793. Zbl 364.16012.

[3] _ On commutativity of periodic rings and near-rings, Acta Math. Acad. Sci. Hungar. 36 (1980), no. 3-4, 293-302. MR 82h:16025. Zbl 464.16026.

[4] _ A commutativity theorem for periodic rings, Math. Japon. 33 (1988), no. 5, 661-662. MR 90a:16027. Zbl 661.16028.

[5] N. Jacobson, Structure of Rings, rev. ed., American Mathematical Society Colloquium Publications, vol. 37, American Mathematical Society, Providence, R.I., 1964. MR 36\#5158.

[6] M. S. Putcha and A. Yaqub, Rings with constraints on nilpotent elements and commutators, Studia Sci. Math. Hungar. 12 (1977), no. 1-2, 193-197. MR 81h:16058. Zbl 437.16021.

AdIL YAQUB: DePARTMENT OF MATHEMATICS, UniVERSITY OF CALIFORNIA, SANTA BARBARA, CA 93106, USA 


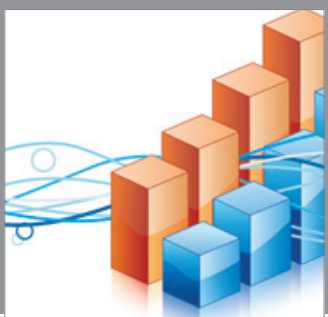

Advances in

Operations Research

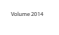

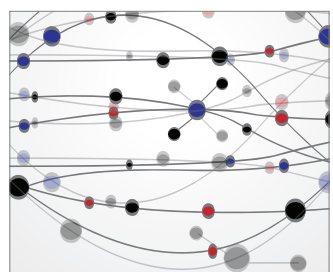

\section{The Scientific} World Journal
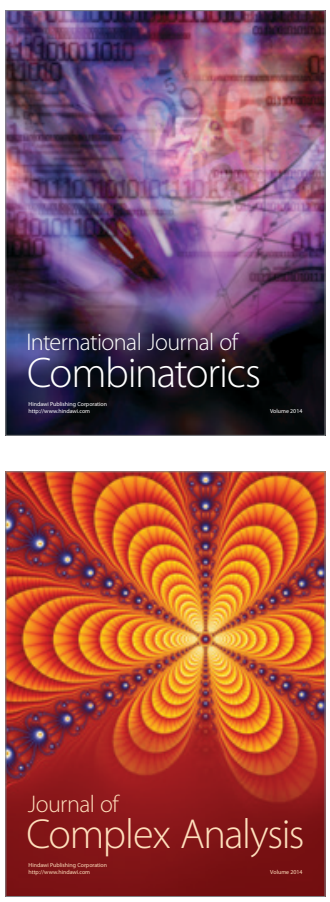

International Journal of

Mathematics and

Mathematical

Sciences
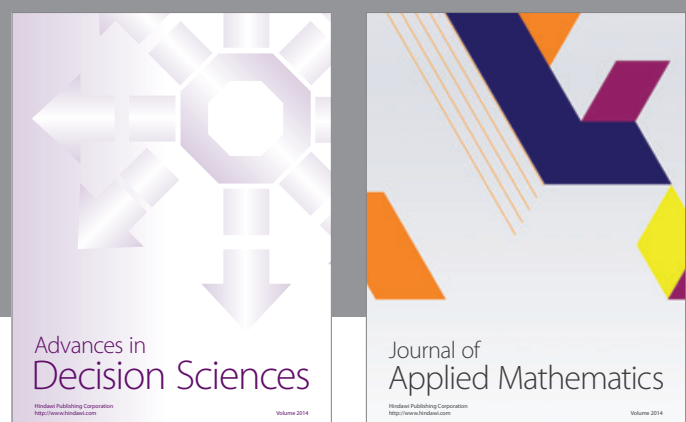

Journal of

Applied Mathematics
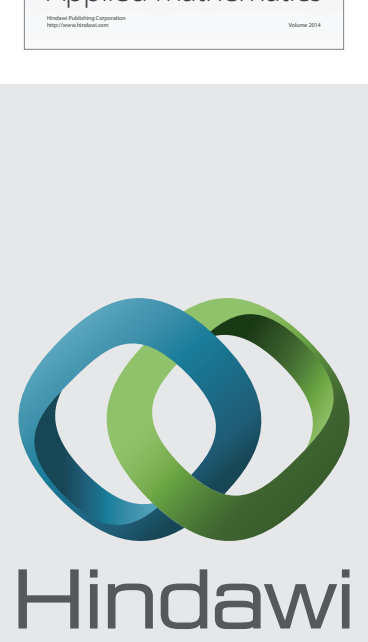

Submit your manuscripts at http://www.hindawi.com
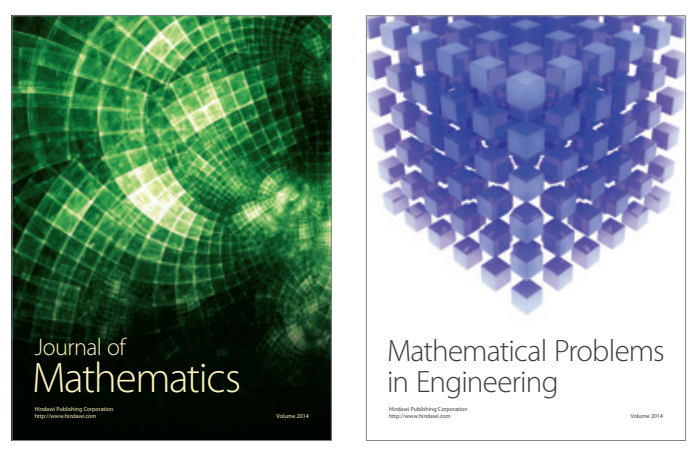

Mathematical Problems in Engineering
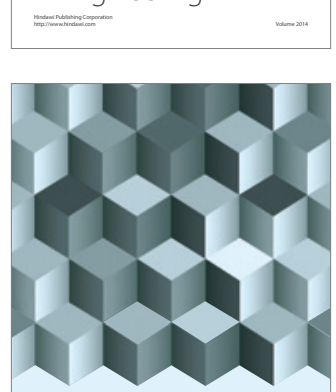

Journal of

Function Spaces
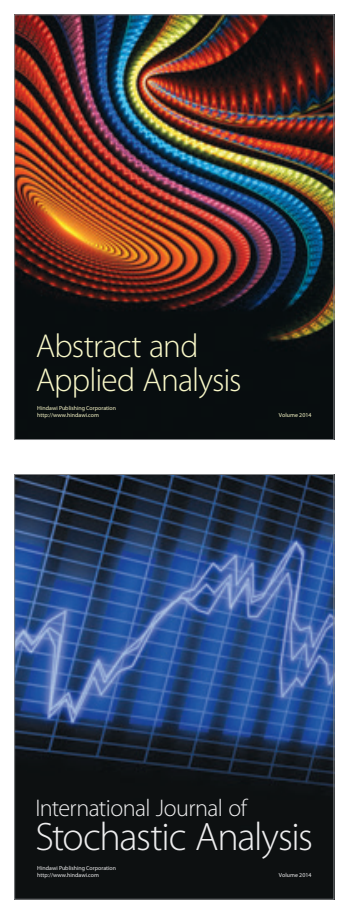

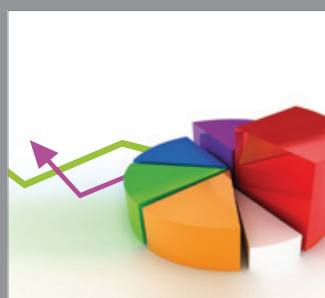

ournal of

Probability and Statistics

Promensencen
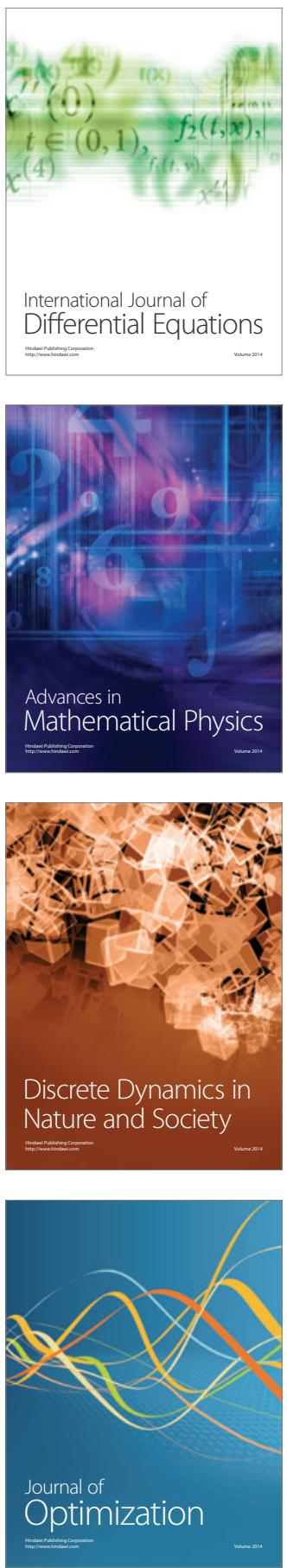